\title{
Comportamiento de un cemento portland resistente a los sulfatos frente al agua desionizada $y$ a disoluciones de sulfato de sodio y de sulfato de magnesio (*)
}

\author{
Comportement d'un ciment portland résistant \\ aux sulfates face a l'eau deionisée et les solutions \\ de sulfate de sodium et de sulfate de magnésium
}

M.a FRANCISCA BERMEJO-MUÑOZ, JOSE LUIS SAGRERA-MORENO y DEMETRIO GASPAR-TEBAR
IETCC/CSIC

Fecha de Recepción: 1-VIII-87

(Madrid, Spain)

\section{RESUMEN}

En este trabajo se estudia el comportamiento de un cemento portland industrial resistente a los sulfatos cuando los lechos granulados -fabricados con dicho cemento, una vez hidratado y conservado durante 7 y 28 días en una cámara húmeda a $21 \pm 2^{\circ} \mathrm{C}$ y con una humedad relativa superior al $90 \%$ - se someten a la acción del agua desionizada, de una disolución de sulfato de sodio y de otra disolución de sulfato de magnesio habiendo determinado:

a. la evolución de los contenidos de los iones $\mathrm{Ca}$ (II) y $\mathrm{SO}_{4}$ (II) en los dos primeros casos, y además $\mathrm{Mg}(\mathrm{II})$, en el tercero, en las disoluciones que han atravesado los lechos de cemento, asi como la del $\mathrm{pH}$ y de la conductividad,

b. la variación del contenido de los iones mencionados en los compuestos correspondientes del cemento de los lechos, una vez sometidos a la acción de las disoluciones agresivas, asi como las modificaciones estructurales experimentadas, $y$

c. las características estructurales de la nueva fase sólida formada en las fracciones recogidas de la disolución de sulfato de magnesio, que han atravesado los lechos de cemento.

En estos trabajos se ha puesto de manifiesto que la cantidad de $\mathrm{Ca}(\mathrm{OH})_{2}$ disuelta (extraída) tanto por el agua desionizada, como por la disolución de sulfato de sodio, disminuye exponencialmente a medida que aumenta el volumen de estas disoluciones, que han atravesado los lechos de cemento, alcanzando un valor asintótico; este fenómeno influye en el avance de las reacciones de hidratación y en la degradación de los compuestos del cemento.

En el caso de la disolución de sulfato de magnesio, se produce un intercambio entre los iones $\mathrm{Ca}$ (II) del $\mathrm{Ca}(\mathrm{OH})_{2}$ del cemento hidratado y el $\mathrm{Mg}$ (II) de la disolución, el cual precipita como brucita en el lecho - llegando a colmatarlo en determinados casos-y en las fracciones de la disolución que han atravesado los lechos. La disolución del $\mathrm{Ca}(\mathrm{OH})_{2}$ se ve influenciada por la presencia de $\mathrm{Mg}(\mathrm{II})$.

\begin{abstract}
RESUME
Dans ce travail on étudie le comportement d'un ciment portland industriel résistant aux sulfates lorsque les lits granulés, fabriqués avec ce ciment une fois hydraté et conservé en armoire humide à $21 \pm 2^{\circ} \mathrm{C}$ et à une hygrométrie supérieure à $90 \%$ pendant 7 et 28 jours, sont soumis à l'action de l'eau deionisée, d'une solution de sulfate de sodium et d'une solution de sulfate de magnésium, ayant déterminé:
\end{abstract}

a. l'evolution de la teneur des ions $\mathrm{Ca}$ (II) et $\mathrm{SO}_{4}$ (II) dans les deux premiers solutions et, en plus, $\mathrm{Mg}$ (II) dans le troisième, qui ont traversé les lits de ciment, ainsi que celle de $\mathrm{pH}$ et de la conductivité,

b. la variation de la teneur des ions mentionées des composés correspondants du ciment hydraté des lits, ainsi que les modifications structurelles expérimentées, et

c. les caracteristiques structurelles de la nouvelle phase solide qui est formée dans les fractions diverses recueillies de la solution de sulfate de magnésium, qui ont traversé les lits.

Dans ces travaux, on a mis en relief que la quantité de $\mathrm{Ca}(\mathrm{OH})_{2}$ dissoute (extraite) aussi bien par l'eau deionisée que par la solution de sulfate de sodium diminue exponentiellement à mesure que le volume de ces solutions, qui ont traversé les lits augment, atteignant une valeur assymtotique; ce fait à une influence à l'avance des réactions d'hydratation et à la dégradation des composés du ciment.

Dans le cas de la solution de sulfate de magnésium, un échange ionique se produit entre les ions $\mathrm{Ca}$ (II) du $\mathrm{Ca}(\mathrm{OH})_{2}$ du ciment hydraté et $\mathrm{Mg}(\mathrm{II})$ de la solution, le quel précipite comme brucite dans les lits -arrivant à le combler dans des cas détérminés- et dans les fractions de la solution qui ont traversé les lits. La solubilité du $\mathrm{Ca}(\mathrm{OH})_{2}$ est influencée par la presence de $\mathrm{Mg}$ (II) à la solution.

(*) Presentado al " $8 .^{\circ}$ congrès international de la chimie des ciments"; Rio de Janeiro, 22-27 septiembre, 1986. 


\section{INTRODUCCION}

El comportamiento de los morteros y hormigones sometidos a la acción de diferentes medios agresivos ha sido objeto de numerosos trabajos como consecuencia del gran número de obras construídas en dichos medios, siendo un tema que ha preocupado y preocupa no sólo desde un punto de vista constructivo, sino científico, para conocer las causas que producen el ataque químico; de aquí, que con objeto de ampliar el conocimiento del mecanismo de las reacciones que tienen lugar cuando los cementos portland hidratados, y sobre todo de sus mezclas con adiciones naturales y artificiales, se someten a la acción de disoluciones agresivas se haya realizado este trabajo, que forma parte de un Programa de Investigación del IETcC a desarrollar a largo plazo, tanto a escala de laboratorio como real.

En otros trabajos (1) (2) (3) (4) se estudia el comportamiento mecánico-resistente de un cemento (cemento 2), análogo al de este trabajo, cuando diversas series de probetas de mortero y de hormigón se someten a la acción de distintas disoluciones agresivas (1) (2), la variación de la concentración iónica del agua de mar en donde han estado sumergidas las probetas de mortero hechas con dos cementos portland industriales (cemento 1 y cemento 2), uno análogo al de este trabajo, y de sus mezclas con una escoria (3) y la evolución de las características estructurales de los compuestos cristalinos de la fracción enriquecida (cemento hidratado-atacado) extraída de una de las probetas de mortero hechas con tres cementos portland industriales (de alta resistencia inicial, resistente a los sulfatos y ordinario, cementos 1 , 2 y 3 , respectivamente), uno es análogo al de este trabajo, y de sus mezclas con escoria, sumergidas en agua de mar artificial, así como de la fase sólida formada en el agua de mar mencionada (4).

\section{PARTE EXPERIMENTAL}

En este trabajo se estudia el comportamiento de un cemento portland resistente a los sulfatos cuando los lechos fabricados con dicho cemento, una vez que se ha hidratado y curado durante 7 ó 28 días en un recinto estanco con una humedad relativa superior al $90 \%$ y a una temperatura de $21 \pm 2^{\circ} \mathrm{C}$, se someten a la acción de agua potable desionizada (sistemas cemento 2 hidratadoagua desionizada), de una disolución de sulfato de sodio (sistemas cemento 2 hidratadodisolución de sulfato de sodio) y de una disolución de sulfato de magnesio (sistemas cemento 2 hidratado-disolución de sulfato de magnesio), determinando la variación de la

\section{INTRODUCTION}

Le comportement de mortiers et bétons soumis à l'action de différentes ambiances agressives à fait l'objet de nombreux travaux comme conséquence du grand nombre d'ouvrages construits dans ces milieux, ce sujet ayant préocupé non seulement d'un point de vue constructif, mais encore scientifique, pour connaître les causes qui produisent l'attaque chimique; c'est ainsi que, à l'objet d'élargir la connaissance du mécanisme des réactions qui ont lieu lorsque les ciments portland hydratés et surtout de ses mélanges avec des ajouts naturels et artificiels, sont soumis à l'action de solutions agressives, ce travail, qui fait partie d'un Projet de Recherche à long terme, a été réalisé aussi bien à échelle de laboratoire comme à échelle réelle.

Dans d'autres travaux (1) (2) (3) (4) nous étudions le comportement mécanique-résistant d'un ciment (ciment 2), analogue à celui qui fait l'objet de ce travail, lorsque diverses séries d'éprouvettes de mortier et de béton sont soumises à l'action de différentes solutions agréssives (1) (2), la variation de la concentration ionique de l'eau de mer où les éprouvettes de mortier fabriquées avec deux ciments portland industriels (ciment 1 et ciment 2) ont été immergées, et des ses mélanges avec laitier (3), l'évolution des caractéristiques structurelles des composants cristallins de la fraction enrichie (ciment hydraté-attaqué) extraite de l'une des éprouvettes de mortier faites avec trois ciments portland industriels (à haute résistance initiale, résistant aux sulfates et normal, ciments 1, 2 et 3, respectivement), et de ses mélanges avec laitier, immergées en eau de mer artificielle, ainsi que de la phase solide formée dans cette eau de mer (4).

\section{PARTIE EXPERIMENTALE}

Dans ce travail nous étudions le comportement d'un ciment portland résistant aux sulfates lorsque les lits fabriqués avec ce ciment, qui s'est hydraté et qui a été conservé pendant 7 ou 28 jours dans chambre humide avec une humidité relative supérieure à $90 \%$ et une température de $21 \pm 2^{\circ} \mathrm{C}$, sont soumis à l'action d'eau déionisée (systèmes ciment 2 hydraté-eau déionisée), d'une solution de sulfate de sodium (systèmes ciment 2 hydratésolution de sulfate de sodium) et d'une solution de sulfate de magnésium (systèmes ciment 2 hydraté-solution de sulfate de magnésium), determinant la variation de la concentration d'ions $\mathrm{Ca}$ (II) et $\mathrm{SO}_{4}$ (II) dans les 
concentración de los iones $\mathrm{Ca}$ (II) y $\mathrm{SO}_{4}$ (II) en los dos primeros sistemas y de los iones $\mathrm{Ca}$ (II), $\mathrm{Mg}$ (II) y $\mathrm{SO}_{4}$ (II) en el tercer sistema tanto de las disoluciones como del cemento hidratado del lecho, la evolución del $\mathrm{pH}$ y de la conductividad de dichas disoluciones, así como las modificaciones estructurales experimentadas por el cemento hidratado del lecho.

\subsection{Materiales utilizados}

\subsubsection{Cemento}

Se ha utilizado un cemento portland industrial resistente al yeso (cemento 2), de fabricación española, cuya composición química y potencial calculada (Bogue), así como la superficie específica, se encuentran en la tabla 1. En la figura 1 se incluyen los DRX de las fases cristalinas correspondientes a los aluminatos del cemento anhidro y del residuo en ácido salicílico-metanol.

\subsubsection{Disoluciones agresivas}

Se han utilizado las siguientes disoluciones:

a. Agua desionizada,

b. Disolución de sulfato de sodio $(2,1 \mathrm{~g} / \mathrm{l}$ de $\mathrm{Na}_{2} \mathrm{SO}_{4}$ ), con una concentración de deux premiers systèmes, et des ions $\mathrm{Ca}$ (II), Mg (II) et $\mathrm{SO}_{4}$ (II) dans le troisième système aussi bien dans les solutions comme dans le ciment hydraté du lit, l'évolution $\mathrm{du} \mathrm{pH}$ et de la conductivité de ces solutions, ainsi que les modifications structurelles expérimentées par le ciment hydraté du lit.

\subsection{Matériaux employés}

\subsubsection{Ciment}

Nous avons utilisé un ciment portland industriel résistant aux sulfates (ciment 2), de fabrication espagnole, dont la composition chimique et potentielle calculée (Bogue), ainsi que la surface spécifique, se trouvent dans le tableau 1. Dans la fig. 1 sont indiqués les DRX des phases cristallines correspondantes aux aluminates du ciment anhydre et du résidu en ácide salicylique-méthanol.

\subsubsection{Solutions agressives}

Nous avons utilisé les solutions suivantes:

a. Eau déionisée.

b. Solution de sulfate de sodium $(2,1 \mathrm{~g} / \mathrm{l}$ de $\mathrm{Na}_{2} \mathrm{SO}_{4}$ ), avec une concentration de

TABLA 1

TABLEAU 1

\begin{tabular}{|c|c|}
\hline $\begin{array}{l}\text { 1. Análisis químico } \\
\text { Analyse chimique }\end{array}$ & $\%$, e.p. \\
\hline P.F. ........ & 1,1 \\
\hline R.I. & 0,2 \\
\hline $\mathrm{SiO}_{2} \ldots \ldots \ldots \ldots \ldots$ & 22,0 \\
\hline $\mathrm{Fe}_{2} \mathrm{O}_{3} \ldots \ldots$ & 4,1 \\
\hline $\mathrm{Al}_{2} \mathrm{O}_{3} \ldots$ & 1,8 \\
\hline $\mathrm{CaO} \ldots .$. & 67,9 \\
\hline $\mathrm{MgO} \ldots \ldots$ & 0,5 \\
\hline $\mathrm{SO}_{3} \ldots \ldots \ldots$ & 2,3 \\
\hline $\mathrm{CaO}$ libre & 1,8 \\
\hline $\begin{array}{l}\text { 2. Composición potencial (Bogue) } \\
\text { Composition potentielle (Bogue) }\end{array}$ & $\%$, e.p. \\
\hline $\mathrm{C}_{3} \mathrm{~S}$. & 75,6 \\
\hline $\mathrm{C}_{2} \mathrm{~S}$. & 6,1 \\
\hline $\mathrm{C}_{3} \mathrm{~A}$. & 0,0 \\
\hline $\mathrm{C}_{4} \mathrm{AF}$ & 10,8 \\
\hline $\begin{array}{l}\text { 3. Superficie especifica (Blaine) } \\
\text { Surface spécifique (Blaine) }\end{array}$ & $3.553 \mathrm{~cm}^{2} / \mathrm{g}$ \\
\hline
\end{tabular}


$\mathrm{SO}_{4}(\mathrm{II})=1,42 \mathrm{~g} / \mathrm{I}<>1,48 \times 10^{-2}$

moles/litro, determinada experimentalmente,

c. Disolución de sulfato de magnesio $(2,76 \mathrm{~g} / \mathrm{l}$ de $\mathrm{MgSO}_{4} .7 \mathrm{H}_{2} \mathrm{O}$ ), con una concentración de $\mathrm{SO}_{4}(\mathrm{II})=1,076 \mathrm{~g} / \mathrm{l}<>1,12 \times 10^{-2}$ moles/litro y de $\mathrm{Mg}$ (II) $=0,272 \mathrm{~g} / \mathrm{l}<>$ $<>1,12 \times 10^{-2}$ moles/litro, determinadas experimentalmente.
$\mathrm{SO}_{4}(\mathrm{II})=1,42 \mathrm{~g} / \mathrm{l}<>1,48 \times 10^{-2}$ moles/litre, déterminée expérimentalement.

c. Solution de sulfate de magnésium $(2,76 \mathrm{~g} / \mathrm{l}$ de $\mathrm{MgSO}_{4} .7 \mathrm{H}_{2} \mathrm{O}$ ), avec une concentration de $\mathrm{SO}_{4}(\mathrm{II})=1,076 \mathrm{~g} / \mathrm{l}<>1,12 \times 10^{-2}$ moles/litre et de $M g(I I)=0,272 \mathrm{~g} / \mathrm{l}<>$ $<>1,12 \times 10^{-2}$ moles/litre, déterminées expérimentalement.
Cemento 2 anhidro
Cemento 2 RSM
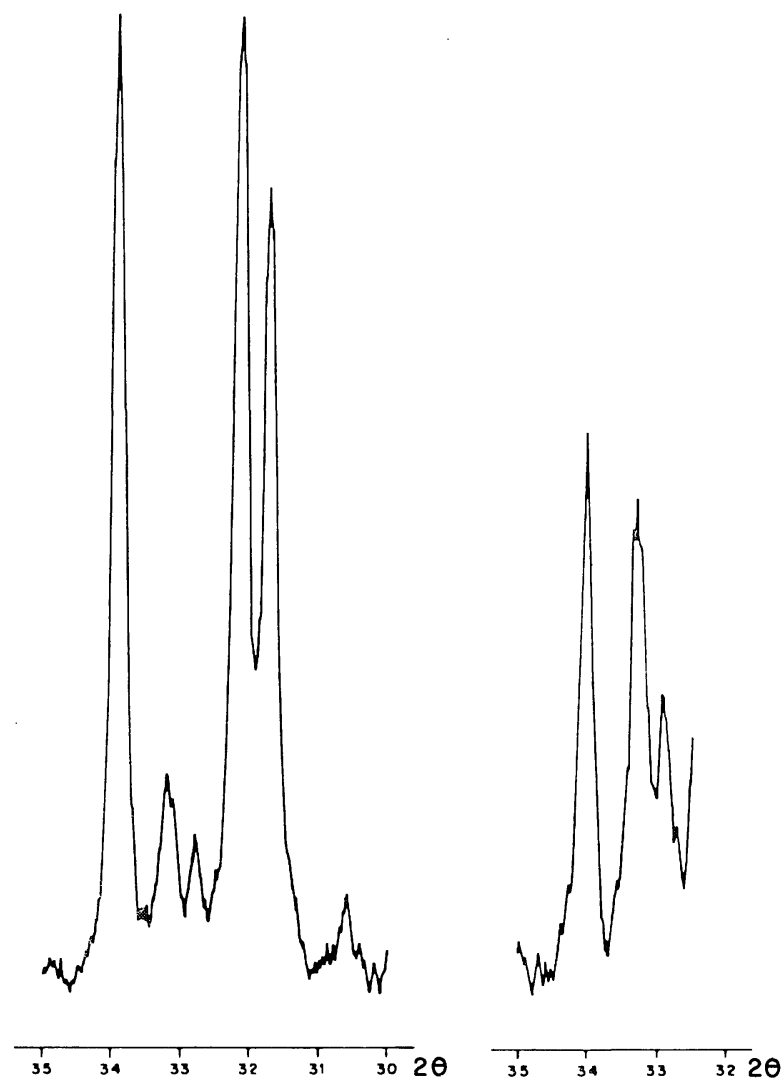

Fig. 1.-DRX del cemento 2 anhidro y del residuo de dicho cemento anhidro atacado con ácido salicílicometanol (RSM).

Fig. 1.-Ciment 2 anhidre et RSM. DRX.

\subsection{Preparación de los lechos de cemento hidratado}

El cemento se amasa con el agua de consistencia, se introduce en una cámara húmeda (humedad relativa $>90 \% ; t^{\circ} \mathrm{C}=$ $=21 \pm 2^{\circ} \mathrm{C}$ ) durante $8 \mathrm{~h}$, se pasa la pasta por un tamiz de $1,5 \mathrm{~mm}$ (luz de malla), obteniendo un granulado que se conserva durante 7 ó 28 días en la cámara húmeda. Se toman $70 \mathrm{~g}$ de la pasta granulada de cemento, que se

\subsection{Préparation des lits de ciment hydraté}

Le ciment est malaxé avec l'eau de consistence, il est introduit en chambre humide (humidité relative $>90 \% ; t^{\circ} \mathrm{C}=$ $=21 \pm 2^{\circ} \mathrm{C}$ ) pendant 8 heures, de suite, on fait passer la pâte par un tamis avec une ouverture de $1,5 \mathrm{~mm}$ obtenant ainsi un granulé de ciment, que l'on introduit dans un tuyeau en plastique $(\varnothing=3,5 \mathrm{~cm} ; h=16 \mathrm{~cm})$, isolée de 
introducen en una columna de plástico $\varnothing=$ $=3,5 \mathrm{~cm} ; \mathrm{h}=16 \mathrm{~cm}$ ), aislada de la atmósfera con nitrógeno.

Las disoluciones, que se encuentran en un depósito nodriza, atraviesan el lecho de cemento, recogiéndose diversas fracciones, en función del tiempo $(0,15-0,16 \mathrm{l} / \mathrm{h})$, en las que se han realizado las determinaciones analíticas mencionadas.

\section{RESULTADOS OBTENIDOS}

Los resultados obtenidos se han representado en las figuras 2 a 10, ambas inclusive.

\section{INTERPRETACION DE RESULTADOS}

4.1. Evolución del contenido de $\mathrm{Ca}$ (II) $\in \mathrm{n}$ los sistemas cemento-agua desionizada y cemento-dis. de $\mathrm{Na}_{2} \mathrm{SO}_{4}$.

En este trabajo se ha puesto de manifiesto que las cantidades de $\mathrm{Ca}(\mathrm{OH})_{2}$ extraídas en los dos sistemas cemento-agua desionizada y cemento-disolución de sulfato de sodio es una función exponencial (figuras 2 y 3), siendo asintótica con relación a la cantidad de disolución que atraviesa el lecho, proceso que influye en el avance de las reacciones de hidratación y en la degradación de los compuestos del cemento, como se puede apreciar en los DRX de las figuras 4 y 5 .

El Ca (II) que aparece en el agua desionizada y en la disolución de $\mathrm{Na}_{2} \mathrm{SO}_{4}$ que han atravesado los lechos de cemento hidratado y granulado procede, fundamentalmente, del $\mathrm{Ca}(\mathrm{OH})_{2}$ formado durante las reacciones de hidratación de los compuestos de la fracción clínker del cemento (dada la solubilidad de los compuestos de calcio del cemento), en virtud de la reacción $(A)$ : l'atmosphère avec l'azote.

Les solutions qui se trouvent dans un réservoir nourrice, traversent le lit de ciment, recueillant diverses fractions, en fonction du temps $(0,15-0,16 \mathrm{l} / \mathrm{h})$, où l'on a effectué les déterminations analytiques mentionnées.

\section{RESULTATS OBTENUS}

Les résultats obtenus ont été représentés dans les figs. 2 à 10, celles-ci étant inclues.

\section{INTERPRETATION DES RESULTATS}

\subsection{Evolution de la teneur en $\mathrm{Ca}$ (II) dans les systèmes ciment-eau déionisée et ciment-sol. de $\mathrm{Na}_{2} \mathrm{SO}_{4}$}

Dans ce travail il a été mis en évidence que les quantités de $\mathrm{Ca}(\mathrm{OH})_{2}$ extraites des deux systèmes ciment-eau déionisée et ciment-solution de sulfate de sodium est une fonction exponentielle (figs. 2 et 3), étant asymptote par rapport à la quantité de solution qui traverse le lit, procès qui influence la progression des réactions d'hydratation et la dégradation des composants du ciment, comme on peut l'apprécier dans les $D R X$ des figures 4 et 5 .

Le Ca (II) qui apparait dans l'eau déionisée et dans la solution de $\mathrm{Na}_{2} \mathrm{SO}_{4}$ qui ont traversé les lits de ciment hydraté et granulé procède, fondamentalement, du $\mathrm{Ca}(\mathrm{OH})_{2}$ formé pendant les réactions d'hydratation des composants de la fraction clinker du ciment (étant donné la solubilité des composants de calcium du ciment), en vertu de la réaction (A):

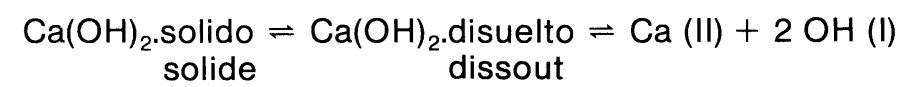

produciendo un incremento brusco del $\mathrm{pH}$ (alcanza valores superiores a 12 en el caso del agua desionizada y a 11,5 de la disolución de $\mathrm{Na}_{2} \mathrm{SO}_{4}$ ). La solubilidad del $\mathrm{Ca}(\mathrm{OH})_{2}$ en agua es $1,230 \mathrm{~g} / \mathrm{l}$, a $20^{\circ} \mathrm{C}$, (5) ó $1,230 \mathrm{~g} / \mathrm{l}$ como $\mathrm{CaO}<>1,625 \mathrm{~g} / \mathrm{l}$ como $\mathrm{Ca}(\mathrm{OH})_{2}(6)$; los silicatos de calcio hidratados son poco solubles en agua, $50 \mathrm{mg} / \mathrm{l}$ como $\mathrm{CaO}<>(7)$ (8) $<>66 \mathrm{mg} / \mathrm{l} \mathrm{como} \mathrm{Ca}(\mathrm{OH})_{2}$; la ettringita se disuelve ligeramente, según ciertos autores (9) (10) y es extraordinariamente insoluble, según otros (11). produisant un accroissement brusque $d u p H$ (il atteint des valeurs supérieures à 12 dans le cas de l'eau déionisée et à 11,5 de la solution de $\mathrm{Na}_{2} \mathrm{SO}_{4}$ ). La solubilité du $\mathrm{Ca}(\mathrm{OH})_{2}$ en eau est de $1,230 \mathrm{~g} / \mathrm{l}$, à $20^{\circ} \mathrm{C}$, (5) ou de $1,230 \mathrm{~g} / \mathrm{l}$ comme CaO $<>1,625 \mathrm{~g} / \mathrm{l}$ comme $\mathrm{Ca}(\mathrm{OH})_{2}(6)$; les silicates de calcium hydratés sont peu solubles dans l'eau, $50 \mathrm{mg} / \mathrm{l} \mathrm{comme} \mathrm{CaO} \mathrm{(7)}$ (8) $<>66 \mathrm{mg} / \mathrm{l} \mathrm{comme} \mathrm{Ca}(\mathrm{OH})_{2}$; l'ettringite se dissout légèrement, d'après certains auteurs (9) (10) et elle est extrèment insoluble, d'après les autres (11). 


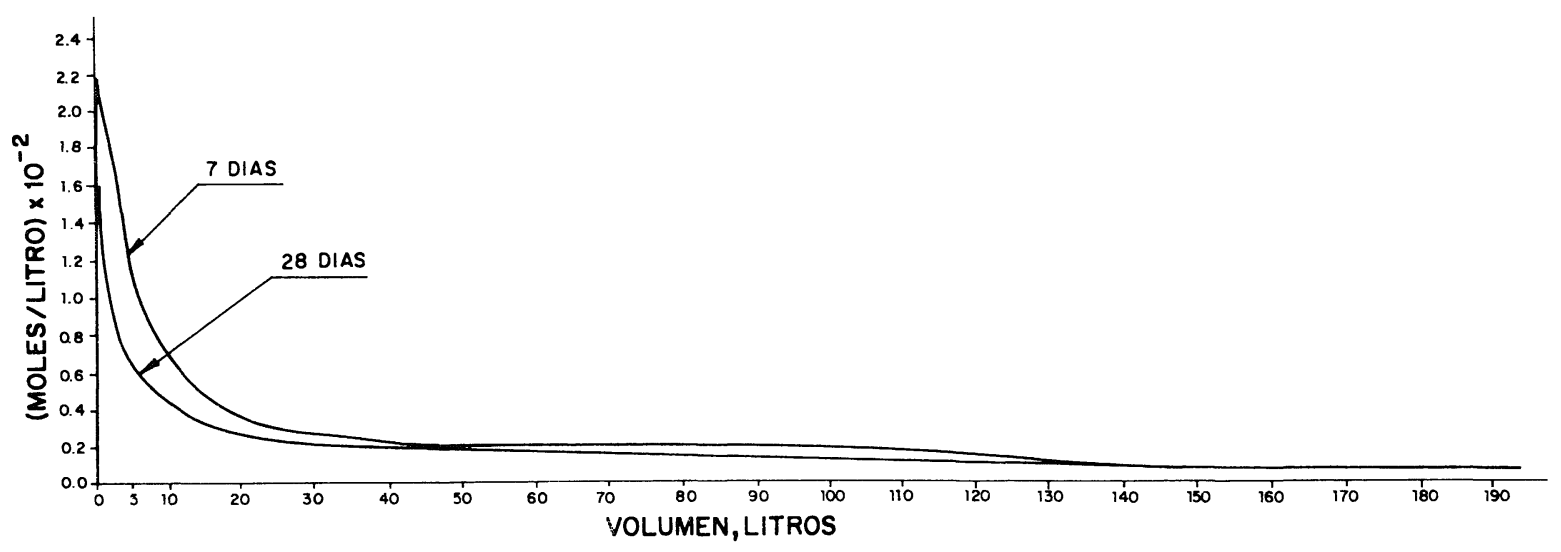

Fig. 2.-Sistemas: cemento 2 hidratado (7-28 dias)-agua desionizada. Evolución del contenido de $\mathrm{Ca}$ (II).

Fig. 2.-Systèmes: ciment 2 hydraté ( 7 et 28 jours)-eau deionisée. Evolution de la teneur de Ca (II).

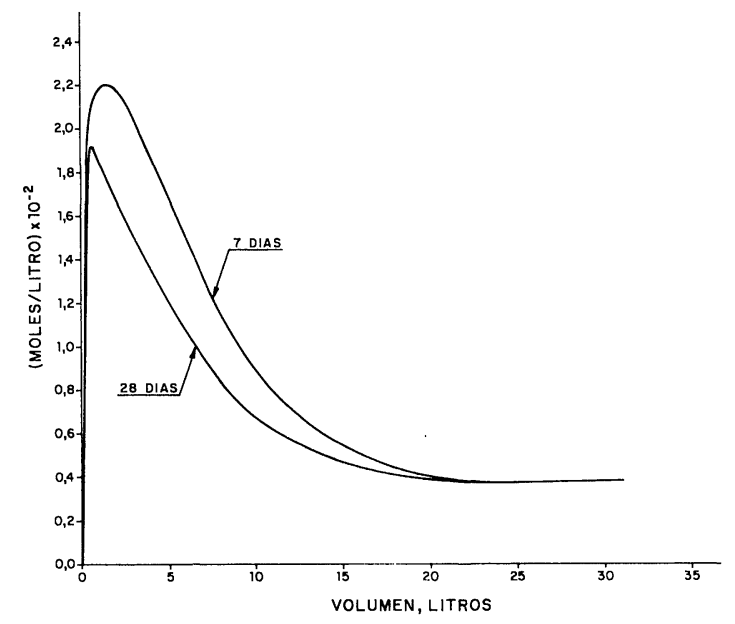

Fig. 3.-Sistemas: cemento 2 hidratado (7 y 28 dias)-disolución de sulfato de sodio.

Evolución del contenido de $\mathrm{Ca}$ (II).

Fig. 3.-Systèmes: ciment 2 hydraté ( 7 et 28 jours)-sol. $\mathrm{Na}_{2} \mathrm{SO}_{4}$.

Evolution de la teneur de Ca (II).

Las concentraciones máximas de $\mathrm{Ca}$ (II) en las disoluciones $\left(2,1 \times 10^{-2}\right.$ moles/litro y $1,6 \times 10^{-2}$ moles/litro) $\left(2,1 \times 10^{-2}\right.$ moles/litro y $1,93 \times 10^{-2}$ moles/litro), asi como los valores del $\mathrm{pH}$ y de la conductividad, se han conseguido cuando han atravesado los lechos
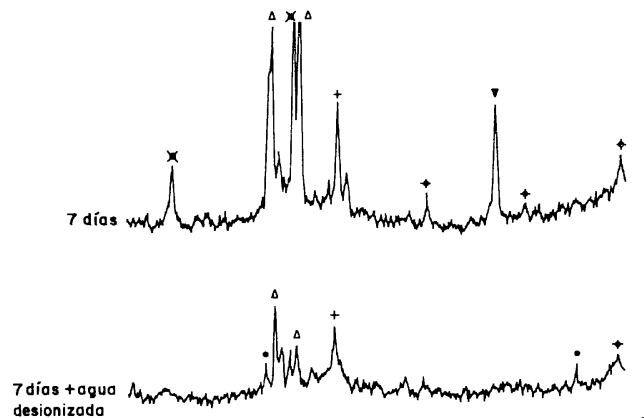

+ CALCITA
+ ETTRINGITA Co(OH) $)_{2}$
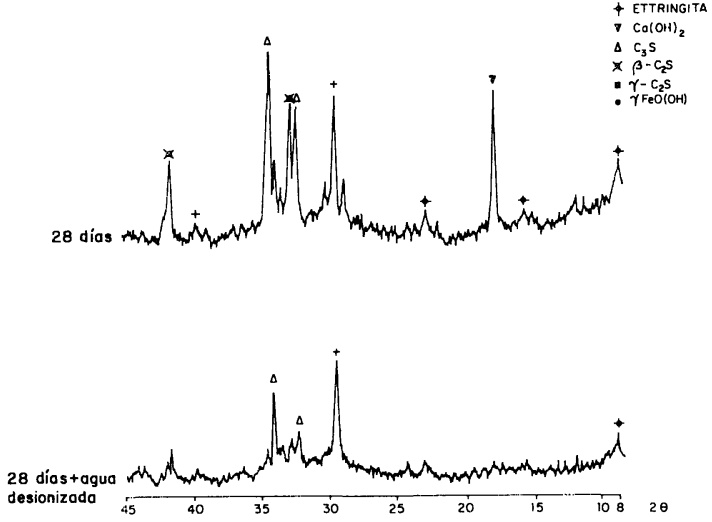

Fig. 4.-Sistemas: cemento 2 hidratado (7 y 28 días)agua desionizada.

DRX del cemento 2 hidratado (7 y 28 días).

DRX del cemento 2 hidratado ( 7 y 28 días) + agua desionizada.

Fig. 4.-Systèmes: ciment 2 hydraté ( 7 et 28 jours)-eau deionisée.

Ciment 2 hydraté ( 7 et 28 jours). DRX.

Ciment 2 hydraté ( 7 et 28 jours) + eau deionisée. DRX.

Les concentrations maximales de $\mathrm{Ca}$ (II) dans les solutions $\left(2,1 \times 10^{-2}\right.$ moles/litre et $1,6 \times 10^{-2}$ moles/litre) $\left(2,1 \times 10^{-2}\right.$ moles/litre et $1,93 \times 10^{-2}$ moles/litre), ainsi que les valeurs du $\mathrm{pH}$ et de la conductivité, ont été obtenues lorsqu'elles ont traversé les lits 0,60 I d'eau 

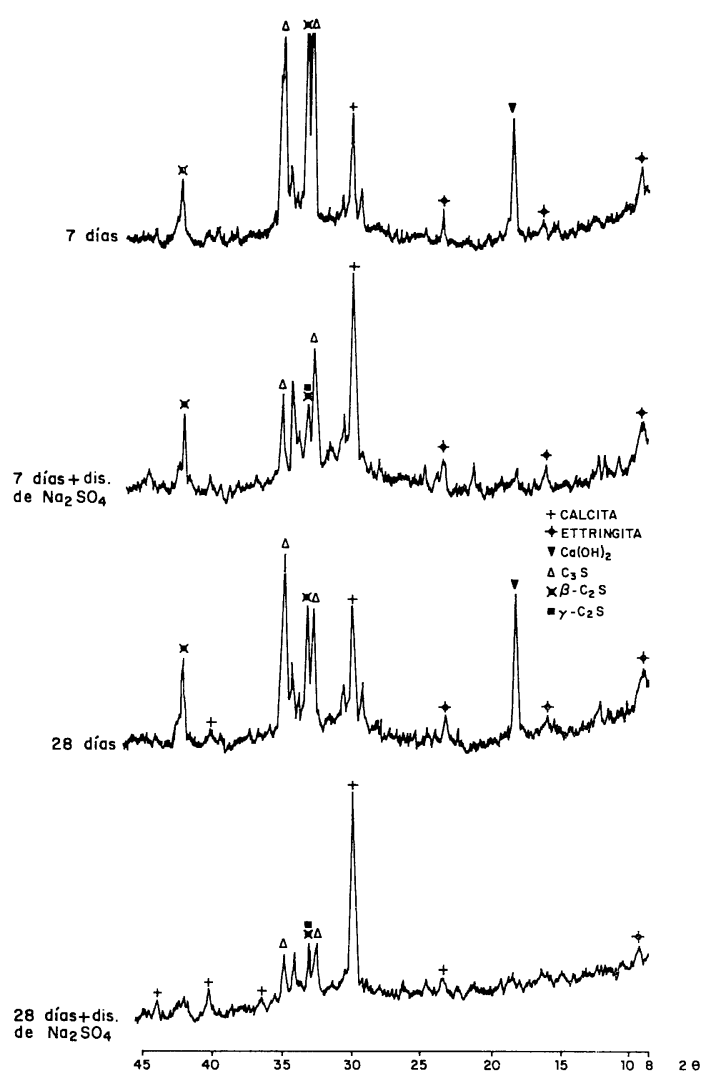

Fig. 5.-Sistemas: cemento 2 hidratado (7 y 28 días)-disolución de sulfato de sodio.

DRX del cemento 2 hidratado ( 7 y 28 días).

DRX del cemento 2 hidratado $(7$ y 28 días $)+$ dis. de $\mathrm{Na}_{2} \mathrm{SO}_{4}$.

Fig. 5.-Systèmes: ciment 2 hydraté (7 et 28 jours)-sol. $\mathrm{Na}_{2} \mathrm{SO}_{4}$.

Ciment 2 hydraté ( 7 et 28 jours). DRX

Ciment 2 hydraté ( 7 et 28 jours) + sol. $\mathrm{Na}_{2} \mathrm{SO}_{4}$. DRX.

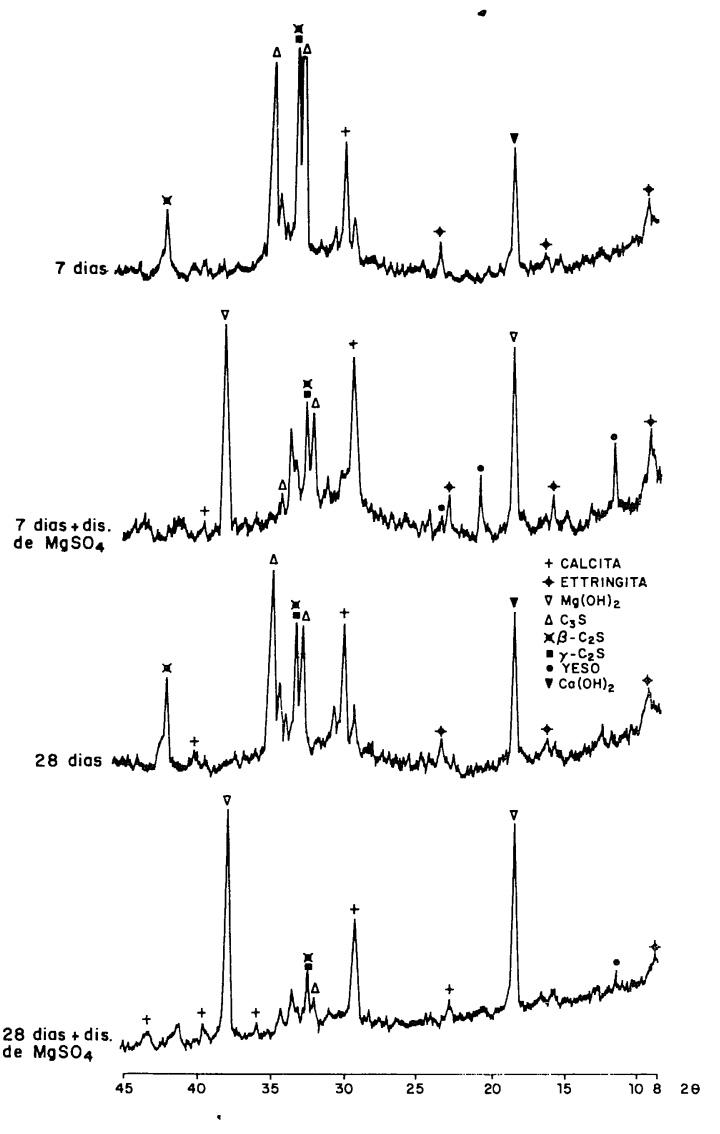

Fig. 6.-Sistemas: cemento 2 hidratado $(7$ y 28 dias)disolución de sulfato de magnesio.

DRX del cemento 2 hidratado (7 y 28 días).

DRX del cemento 2 hidratado $(7$ y 28 días) + dis. de $\mathrm{MgSO}_{4}$.

Fig. 6.-Systèmes: ciment 2 hydraté (7 et 28 jours)-sol. $\mathrm{MgSO}_{4}$.

Ciment 2 hydraté ( 7 et 28 jours). DRX.

Ciment 2 hydraté ( 7 et 28 jours) + sol. $\mathrm{MgSO}_{4}$. DRX:

TABLA 2

Cantidad de Ca (II) en los compuestos; moles, \%

TABLEAU 2

Quantité de Ca (II) dans les composants; moles, \%

\begin{tabular}{|c|c|c|c|c|c|c|}
\hline \multirow{3}{*}{$\begin{array}{l}\text { Compuestos } \\
\text { Composants }\end{array}$} & \multicolumn{6}{|c|}{$\begin{array}{l}\text { Disoluciones } \\
\text { Solutions }\end{array}$} \\
\hline & \multicolumn{2}{|c|}{$\begin{array}{l}\text { Agua desionizada } \\
\text { Eau deionisée }\end{array}$} & \multicolumn{2}{|c|}{$\mathrm{Na}_{2} \mathrm{SO}_{4}$} & \multicolumn{2}{|c|}{$\mathrm{MgSO}_{4}$} \\
\hline & $\begin{array}{l}7 \text { dias } \\
7 \text { jours }\end{array}$ & $\begin{array}{l}28 \text { días } \\
28 \text { jours }\end{array}$ & $\begin{array}{l}7 \text { dias } \\
7 \text { jours }\end{array}$ & $\begin{array}{l}28 \text { días } \\
28 \text { jours }\end{array}$ & $\begin{array}{l}7 \text { días } \\
7 \text { jours }\end{array}$ & $\begin{array}{l}28 \text { días } \\
28 \text { jours }\end{array}$ \\
\hline $\begin{array}{l}\text { Cemento }(100,0) \\
\text { Ciment }\end{array}$ & 30,0 & 46,2 & 57,1 & 67,7 & 52,9 & 74,2 \\
\hline $\begin{array}{l}\text { Disolucion }(0,0) \\
\text { Solution }\end{array}$ & 65,7 & 50,8 & 34,3 & 32,3 & 47,1 & 25,8 \\
\hline $\begin{array}{l}\text { Suma }(100,0) \\
\text { Somme }\end{array}$ & 95,7 & 97,0 & 91,4 & 100,0 & 100,0 & 100,0 \\
\hline
\end{tabular}


0,60 I de agua desionizada para el cemento hidratado 7 días y 0,36 I en el caso del cemento hidratado 28 días; 1,01 litros de la disolución de $\mathrm{Na}_{2} \mathrm{SO}_{4}$ para el cemento hidratado 7 días y 0,49 I para el hidratado 28 dias, respectivamente.

En las extracciones siguientes disminuye exponencialmente, llegando a ser menores de $0,2 \times 10^{-2}$ moles/litro en el primer sistema y de $0,4 \times 10^{-2}$ moles/litro en el segundo.

Las cantidades de $\mathrm{Ca}$ (II) $<>\mathrm{Ca}(\mathrm{OH})_{2}$ extraídas de los lechos de cemento son función del poder disolvente de la disolución y del volumen que ha atravesado los lechos de cemento, por una parte, así como del tiempo de hidratación del cemento, por otra parte, como se puede apreciar en los DRX de las figuras 4 y 5 y en la tabla 2 . Así, dichas cantidades representan el 65,7\% (por 194,69 I) y 50,8 \% (por 140,19 I) para el agua desionizada y $34,3 \%$ (por 26,54 l) y $32,3 \%$ (por 31,94 l) para la disolución de sulfato de sodio (en donde se debe tener en cuenta el efecto salino), según se trate del cemento hidratado durante 7 ó 28 días, respectivamente; el resto queda en las probetas de mortero en forma de sales de calcio; todo el $\mathrm{Ca}(\mathrm{OH})_{2}$ ha desaparecido (DRX de las figuras 4 y 5). Todos estos fenómenos están de acuerdo con el grado de hidratación y carbonatación del cemento, así como con el avance de las reacciones de hidratación.

\subsection{Evolución de los contenidos de $\mathrm{Ca}$ (II) y $\mathrm{Mg}$ (II) en los sistemas cemento-dis. de $\mathrm{MgSO}_{4}$}

En los sistemas cemento-disolución de sulfato de magnesio se producen un conjunto de procesos fisicoquímicos que dan lugar a la formación de compuestos en el cemento de los lechos ( 7 y 28 días), de una nueva fase sólida en la disolución que ha atravesado los lechos, y a la eliminación de otros.

En el cemento de los lechos, como se ha probado por DRX (figura 6), se ha formado brucita y yeso secundario, ha desaparecido la portlandita y han disminuido los compuestos anhidros del cemento; además, se ha incrementado la cantidad de ettringita $y$ calcita.

La disolución que ha atravesado los lechos de cemento ha experimentado modificaciones en lo que se refiere a los contenidos de $\mathrm{Ca}$ (II), $\mathrm{Mg}$ (II) y $\mathrm{SO}_{4}$ (II), así como al valor del $\mathrm{pH}$ y de la conductividad; en su seno se ha formado una nueva fase sólida que está compuesta por déionisée pour le ciment hydraté 7 jours et 0,36 I dans le cas du ciment hydraté 28 jours; 1,01 litres de la solution $\mathrm{Na}_{2} \mathrm{SO}_{4}$ pour le ciment hydraté 7 jours et 0,49 pour 28 jours, respectivement.

Dans les extractions suivantes elles décroissent exponentiellement pouvant être plus petites que $0,2 \times 10^{-2}$ moles/litre dans le premier système et $0,4 \times 10^{-2}$ moles/litre dans le second.

Les quantités de $\mathrm{Ca}(\mathrm{II})<>\mathrm{Ca}(\mathrm{OH})_{2}$ extraites des lits de ciment sont fonction du pouvoir dissolvant de la solution et du volume qui a traversé les lits de ciment, d'une part, ainsi que du temps d'hydratation du ciment, de l'autre, comme on peut l'apprécier dans les DRX des figures 4 et 5 et dans le tableau 2. Ainsi, ces quantités représentent $65,7 \%$ (par 194,69 I) et $50,8 \%$ (par 140,19 l) pour l'eau déionisée et $34,3 \%$ (par 25,64 l) et 32,3\% (par 31,94 I) pour la solution de sulfate de sodium (là on doit tenir compte de l'effet salin), selon qu'il s'agisse du ciment hydraté pendant 7 ou 28 jours, respectivement; le reste, reste dans les éprouvettes de mortier sous forme de sels de calcium; tout le $\mathrm{Ca}(\mathrm{OH})_{2}$ a disparu (DRX des figs. 4 et 5). Tous ces phénomènes sont en rapport avec le degré d'hydratation et de carbonatation du ciment, ainsi qu'avec la progression des réactions d'hydratation.

\subsection{Evolution des teneurs en $\mathrm{Ca}$ (II) et Mg (II) dans les systèmes ciment-sol. de $\mathrm{MgSO}_{4}$}

Dans les systèmes ciment-solution de sulfate de magnésium se produit un ensemble de precessus physicochimiques qui donnent lieu à la formation de composants dans les lits de des lits ( 7 et 28 jours), d'une nouvelle phase solide dans la solution qui a traversé les lits, et à la elimination des autres.

Dans le ciment des lits, il s'est formé brucite et gypse secondaire (prouvé par DRX, fig. 6), la portlandite a disparu et les composants anhydres du ciment ont diminué; et aussi, la quantité d'ettringite et de calcite a augmenté.

La solution qui a traversé les lits de ciment a expérimenté des modifications en ce qui concerne les teneurs en $\mathrm{Ca}$ (II), $\mathrm{Mg}$ (II) et $\mathrm{SO}_{4}(\mathrm{II})$, ainsi que la valeur du $\mathrm{pH}$ et de la conductivité; à leur sein, une nouvelle phase solide s'est formée, composée de brucite et de 
brucita y calcita (DRX de la figura 7), en donde han precipitado, por alcanzarse los Ps correspondientes.

En estos trabajos se ha puesto de manifiesto que el $\mathrm{Mg}$ (II) de la disolución (1,12 × 10-2 moles/litro) desaparece total o parcialmente cuando atraviesa el lecho de cemento hidratado, habiendo precipitado como $\mathrm{Mg}(\mathrm{OH})_{2}$ en el mismo o en la disolución; totalmente, cuando la concentración de Ca (II) en la disolución, extraída del cemento, es $\geq 1,12 \times$ $\times 10^{-2}$ moles/litro y el $\mathrm{pH} \geq 11,5 \mathrm{y}$, parcialmente, cuando ambas características son menores (figuras 8 y 9). El precipitado de $\mathrm{Mg}(\mathrm{OH})_{2}$ en el lecho produce una disminución de la porosidad, llegando a veces a colmatarlo e impidiendo el paso de la disolución (figura 10); este precipitado se inicia en la zona superior de la columna del lecho de cemento, por lo que la parte principal de intercambio debe descender a medida que fluye más disolución de $\mathrm{MgSO}_{4}$.

La formación de brucita en el cemento del lecho tiene lugar según la siguiente reacción: calcite (DRX de la fig. 7) où elles ont précipité, ayant atteint les $P$ s correspondents.

Dans ces travaux il a été mis en évidence que le $\mathrm{Mg}$ (II) de la solution $\left(1,12 \times 10^{-2}\right.$ moles/litre) disparait totalement ou partiellement lorsqu'il traverse le lit de ciment hydraté, hayant précipité comme $\mathrm{Mg}(\mathrm{OH})_{2}$ sur place ou dans la solution; totalement, lorsque la concentration de $\mathrm{Ca}$ (II) dans la solution, extraite du ciment, est $\geq 1,12 \times 10^{-2}$ moles/litre et le $\mathrm{pH} \geq 11,5$ et, partiellement, lorsque les deux caractéristiques sont plus petites (figs. 8 et 9). Le précipité de $\mathrm{Mg}(\mathrm{OH})_{2}$ dans le lit produit une diminution de la porosité, arrivant parfois à se colmater et empêchant le passage de la solution (fig. 10); le précipité commence dans la zone supérieure de la colonne du lit de ciment, raison pour laquelle la partie principale d'échange doit descendre à mesure qu'une plus grande quantité de solution de $\mathrm{MgSO}_{4}$ s'écoule.

La formation de brucite dans le ciment du lit à lieu d'après la réaction suivante:

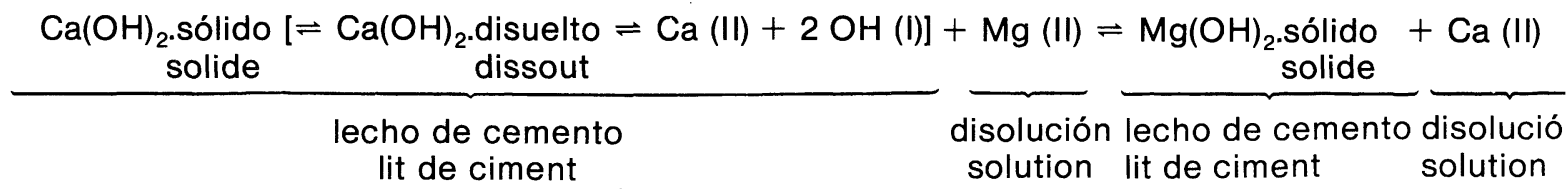

ya que Ps $\left[\mathrm{Mg}(\mathrm{OH})_{2}\right]=1,8 \times 10^{-11}<\mathrm{P}$ 's $\left[\mathrm{Ca}(\mathrm{OH})_{2}\right]=5,5 \times 10^{-6}$, cumpliéndose, por otra parte, $[\mathrm{Mg}(\mathrm{II})][\mathrm{OH}(\mathrm{I})]^{2}>\mathrm{Ps}\left(1,8 \times 10^{-11}\right)$. En este caso, por cada mol de iones $\mathrm{Mg}$ (II) que desaparece de la disolución aparece otro de $\mathrm{Ca}$ (II). puisque Ps $\left[\mathrm{Mg}(\mathrm{OH})_{2}\right]=1,8 \times 10^{-11}<$ $<P^{\prime} \mathrm{S}\left[\mathrm{Ca}(\mathrm{OH})_{2}\right]=5,5 \times 10^{-6}$, s'accomplissant d'autre part, $[\mathrm{Mg}(\mathrm{II})][\mathrm{OH}(\mathrm{I})]^{2}>\mathrm{Ps}\left(1,8 \times 10^{-11}\right)$. En ce cas là, pour chaque mol d'ions $\mathrm{Mg}$ (II) qui disparait de la solution, il en apparait un autre de $\mathrm{Ca}$ (II).

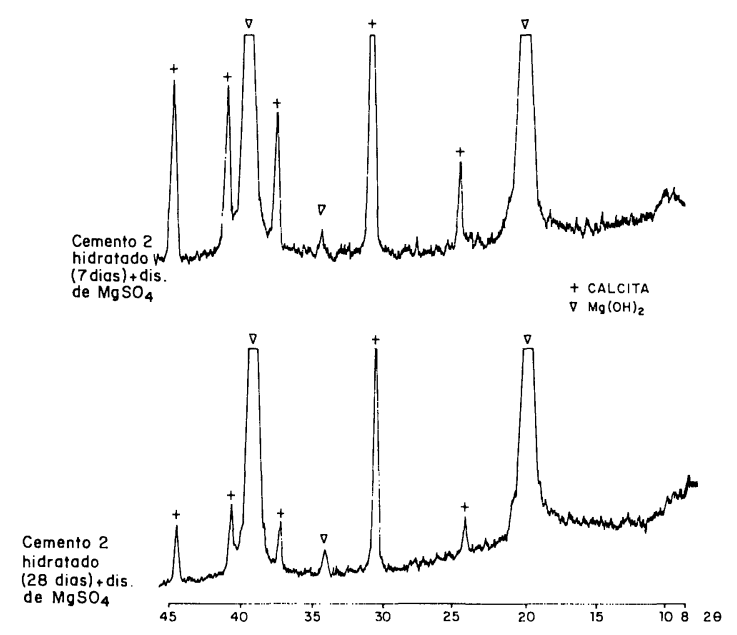

Fig. 7.-Sistemas: cemento 2 hidratado (7 y 28 días)-disolución de sulfato de magnesio. DRX de la nueva fase sólida.
Fig. 7.-Systèmes: ciment 2 hydraté (7 et 28 jours)-sol. $\mathrm{MgSO}_{4}$.

Nouvelle phase solide. DRX. 
$\mathrm{EI} \mathrm{Ca}(\mathrm{OH})_{2}$ del lecho de cemento hidratado se disuelve parcialmente en la disolución de $\mathrm{MgSO}_{4}$, según el equilibrio (A), llegando a desaparecer (DRX de la figura 6); en las primeras extracciones el contenido de $\mathrm{Ca}(\mathrm{II})<>\mathrm{Ca}(\mathrm{OH})_{2}$ disuelto alcanza su valor máximo tanto en el lecho de cemento hidratado 7 días (2,8 $\times 10^{-2}$ moles/litro) como 28 días $\left(2,38 \times 10^{-2}\right.$ moles/litro) cuando han pasado 0,72 I y 0,21 I, respectivamente; a continuación, disminuye alcanzando los

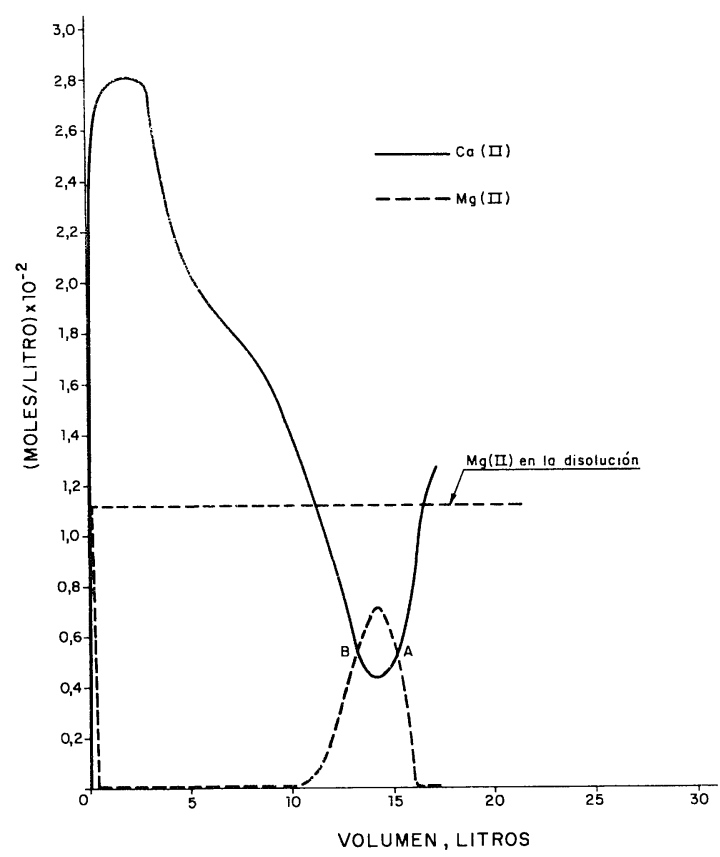

Fig. 8.-Sistema: cemento 2 hidratado (7 días)-disolución de sulfato de magnesio.

Evolución de los contenidos de $\mathrm{Ca}$ (II) y de $\mathrm{Mg}$ (II).

Fig. 8.-Système: ciment 2 hydraté (7 jours)-sol. $\mathrm{MgSO}_{4}$. Evolution des teneurs de $\mathrm{Ca}$ (II) et de $\mathrm{Mg}$ (II).

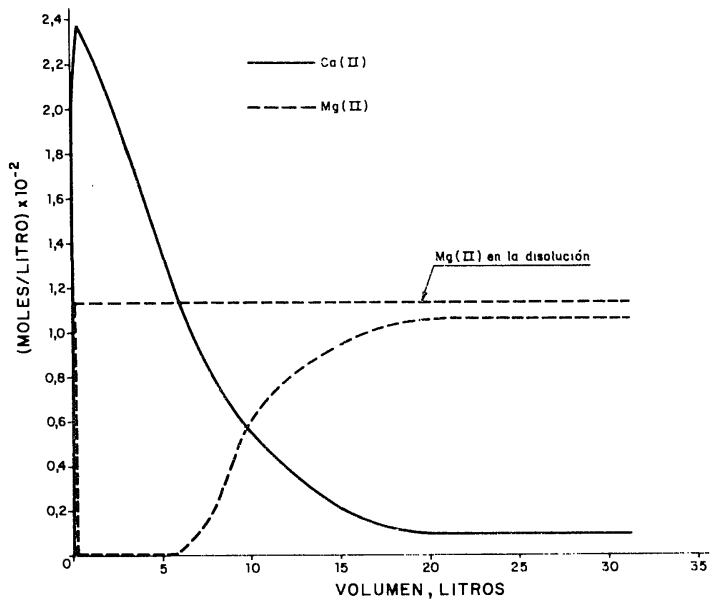

Fig. 9.-Sistema: cemento 2 hidratado (28 días)-disolución de sulfato de magnesio.

Evolución de los contenidos de $\mathrm{Ca}$ (II) y de $\mathrm{Mg}$ (II).

Fig. 9.-Système: ciment 2 hydraté (28 jours)-sol. $\mathrm{MgSO}_{4}$. Evolution des teneurs de $\mathrm{Ca}$ (II) et de $\mathrm{Mg}$ (II).
Le $\mathrm{Ca}(\mathrm{OH})_{2}$ du lit de ciment hydraté, se dissout partiellement dans la solution de $\mathrm{MgSO}_{4}$, d'après l'équilibre (A), arrivant à disparaître (DRX de la fig. 6); dans les premières extractions, la teneur en $\mathrm{Ca}(\mathrm{II})<>\mathrm{Ca}(\mathrm{OH})_{2}$ dissout atteint sa valeur maximale aussi bien dans lè lit de ciment hydraté 7 jours $\left(2,8 \times 10^{-2}\right.$ moles/litre) comme 28 jours $\left(2,38 \times 10^{-2}\right.$ moles/litre) lorsque 0,72 I et 0,21 l,

respectivement, ont coulé; par la suite, il diminue, atteignant les valeurs minimales
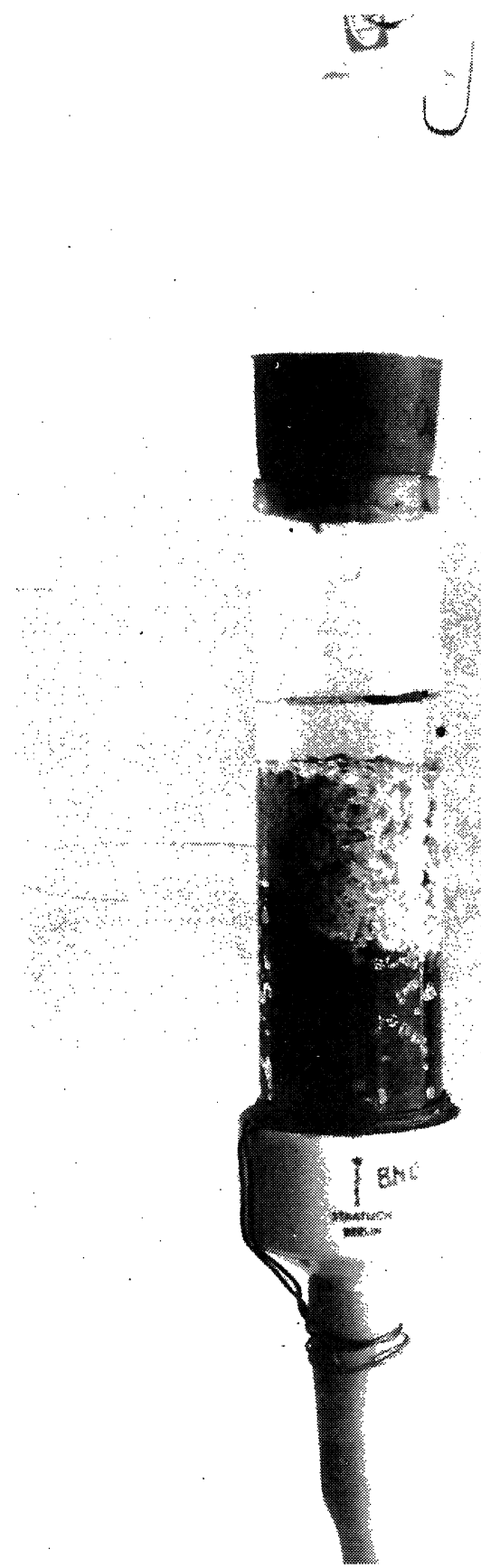

Fig. 10.-Sistema: cemento 2 hidratado (7 días)-disolución de sulfato de magnesio.

Vista del leccho.

Fig. 10.-Système: ciment 2 hydraté (7 jours)-sol. $\mathrm{MgSO}_{4}$ Vue du lit. 
valores mínimos $\left(0,4 \times 10^{-2}\right.$ moles/litro para e! primer lecho, aumentando a continuación, y $0,08 \times 10^{-2}$ moles/litro para el segundo lecho); los contenidos de $\mathrm{Ca}$ (II) siguen la evolución representada en las figuras 8 y 9 , en las que se ha incluído la evolución de los contenidos de $\mathrm{Mg}$ (II).

En estos sistemas se observa que la evolución del contenido de $\mathrm{Mg}$ (II) en las distintas extraciones de la disolución está íntimamente ligada a la de $\mathrm{Ca}$ (II), de tal modo que cuando la eliminación del Mg (II) en la disolución, por precipitación en el lecho de cemento como $\mathrm{Mg}(\mathrm{OH})_{2}$, es parcial se cumple [Ca (II)] + $+[\mathrm{Mg}(\mathrm{II})]=$ cte. $\left(\simeq 1,12 \times 10^{-2}\right.$ moles/litro $=$ = concentración inicial de $\mathrm{Mg}$ (II) en la disolución), de tal modo que por cada mol de $\mathrm{Mg}$ (II) que desaparece de la disolución aparece otro de $\mathrm{Ca}$ (II), como se ha señalado anteriormente. En los puntos de corte de las gráficas que representan la evolución de los contenidos de $\mathrm{Ca}$ (II) y $\mathrm{Mg}$ (II) (figuras 8 y 9), se cumple $[\mathrm{Ca}(\mathrm{II})]=[\mathrm{Mg}$ (II) $]=0,6 \times 10^{-2}$ moles/litro; es decir, se ha conseguido el equilibrio (B). Equilibrio ligado a las reacciones de hidratación del cemento y a la formación de $\mathrm{Ca}(\mathrm{OH})_{2}$, que se ven favorecidas por la eliminación de iones $\mathrm{OH}$ (I) y por la disolución de la portlandita.

Los hechos mencionados prueban que las cantidades de $\mathrm{Ca}(\mathrm{II})<>\mathrm{Ca}(\mathrm{OH})_{2}$ extraídas de los lechos de cemento dependen del tiempo de hidratación del cemento del lecho y de la influencia del cation $\mathrm{Mg}$ (II).

Así, en el caso del lecho de cemento hidratado durante 7 días, el precipitado de $\mathrm{Mg}(\mathrm{OH})_{2}$ ha colmatado a dicho lecho, se ha extraído el $47,1 \%$ (por 17,04 I) (tabla 2), mientras que en el caso del lecho de cemento hidratado durante 28 días se ha extraído el $25,8 \%$ (por 30,62 I) (tabla 2) y se ha depositado el $38,9 \%$ de $\mathrm{Mg}$ (II), debido a la influencia de la precipitación de dicho $\mathrm{Mg}$ (II) que desplaza el equilibrio (B) hacia la formación de brucita por las razones expuestas anteriormente.

En la mayoria de las extracciones ha aparecido una pequeña cantidad de precipitado (del orden de $\mathrm{mg}$ ), que agrupados han permitido determinar su composición química y estudiarlo por DRX (figura 7), habiendo identificado los picos de los compuestos cristalinos calcita y brucita, que han precipitado por darse las condiciones favorables para alcanzar los productos de solubilidad correspondientes.

\subsection{Evolución del contenido de $\mathrm{SO}_{4}$ (II)}

El agua desionizada disuelve, también, a los $\left(0,4 \times 10^{-2}\right.$ moles/litre pour le premier lit, augmentant par la suite, et $0,08 \times 10^{-2}$ moles/litre pour le deuxième lit), les teneurs en Ca (II) suivent l'évolution représentée dans le fig. 8 et 9 , où nous indiquons aussi, l'évolution des teneurs en $\mathrm{Mg}$ (II).

Dans ces systèmes on remarque que l'évolution de la teneur en $\mathrm{Mg}$ (II) dans les différentes extractions de la solution est intimement en rapport à celle de $\mathrm{Ca}(\mathrm{II})$, de telle façon que, lorsque l'élimination du Mg (II) dans la solution, par précipitation dans le lit de ciment comme $\mathrm{Mg}(\mathrm{OH})_{2}$, est partielle, il y a $[\mathrm{Ca}(I I)]+[\mathrm{Mg}(\mathrm{II})]=$ cte. $\left(\simeq 1,12 \times 10^{-2}\right.$ moles/litre $=$ concentration initielle de $M g(I I)$ dans la solution) de telle façon que pour chaque mol de Mg (II) qui disparait de la solution, un autre de Ca (II) apparait, comme il a été signalé auparavant. Dans les points d'intersection des graphiques qui représentent les teneurs en Ca (II) et Mg (II) (figs. 8 et 9), il y a $[\mathrm{Ca}(I I)]=[\mathrm{Mg}(I I)]=0,6 \times 10^{-2}$ moles/litre; c'est à dire, l'équilibre (B) est atteint. Equilibre lié aux réactions d'hydratation du ciment et à la formation de $\mathrm{Ca}(\mathrm{OH})_{2}$, qui se trouvent favorisés par l'élimination d'ions $\mathrm{OH}(\mathrm{I})$ et par la dissolution de la portlandite.

Les faits mentionnés prouvent que les quantités de $\mathrm{Ca}(\mathrm{II})<>\mathrm{Ca}(\mathrm{OH})_{2}$ extraites des lits de ciment dépendent du temps d'hydratation du ciment et de l'influence $d u$ cation $\mathrm{Mg}(\mathrm{II})$.

Ainsi, dans le cas du lit de ciment hydraté pendant 7 jours, le précipité de $\mathrm{Mg}(\mathrm{OH})_{2}$ a colmaté ce lit, dont $47,1 \%$ (pour 17,04 I) a été extrait (tableau 2), tandis que, dans le cas du lit de ciment hydraté pendant 28 jours, 25,8 \% (pour 30,62 I) en a été extrait (tableau 2) et 38,9\% de Mg (II) s'est déposé, à cause de l'influence de la précipitation de ce $M g$ (II) qui déplace l'equilibre (B) vers la formation de brucite pour les raisons dont nous avons fait l'exposé auparavant.

Dans la plûpart des extractions, des petites quantités de précipité sont apparues (de l'ordre de mg) qui, groupés, ont permis de déterminer leur composition chimique et de les étudier par DRX (fig. 7), ayant identifié les pics des composants cristallins calcite et brucite, qui ont précipité ayant les conditions favorables pour atteindre les produits de solubilté correspondants.

\subsection{Evolution de la teneur en $\mathrm{SO}_{4}$ (II)}

L'eau déionisée dissout, aussi, les sulfates 
sulfatos presentes en el cemento, siendo función del grado de hidratación y de formación de nuevos compuestos; así, en el caso del lecho de cemento hidratado durante 7 días quedan en el cemento el $41 \%$-para 194,69 1- de dichos compuestos y en el del lecho de cemento hidratado durante 28 días el $53 \%$, para 140,19 I. La extracción de dichos compuestos disminuye exponencialmente, como en caso del $\mathrm{Ca}$ (II), con el paso del agua desionizada.

Por el contrario, en el caso de la disolución de $\mathrm{Na}_{2} \mathrm{SO}_{4}$, cuando pasan las primeras cantidades de dicha disolución, disminuye la concentración de iones $\mathrm{SO}_{4}$ (II) de la disolución (son retenidos por el lecho de cemento) y, posteriormente, dicha concentración se mantiene prácticamente constante. En resumen, en un principio, parte de la concentración de iones $\mathrm{SO}_{4}$ (II), en pequeña cantidad, y dadas las características estructurales del cemento, es retenida por dicho cemento hidratado del lecho, fundamentaimente, en forma de $\mathrm{CaSO}_{4} \cdot 2 \mathrm{H}_{2} \mathrm{O}$, por tener $\mathrm{Ca}(\mathrm{II})$ - procedente del $\mathrm{Ca}(\mathrm{OH})_{2}-$. Por el contrario, una vez alcanzado el equilibrio y al no existir $\mathrm{Ca}$ (II) $<>\mathrm{Ca}(\mathrm{OH})_{2}$, por haberse disuelto, cesa la formación de dicho compuesto, y de ettringita, actuando la disolución de $\mathrm{Na}_{2} \mathrm{SO}_{4}$ como disolvente de los compuestos del cemento.

En los sistemas cemento-disolución de sulfato de magnesio tiene lugar un proceso análogo al de la disolución de sulfato de sodio [el contenido de $\mathrm{SO}_{4}$ (II) disminuye èn la primera extracción y posteriormente no experimenta variación]; ahora bien, dadas las características de estos sistemas que ponen en juego mayores cantidades de Ca (II), según el equilibrio (B), las cantidades retenidas de iones $\mathrm{SO}_{4}$ (II), son mayores (del orden del 10-12 \%, frente al 6-7\%, con relación a la cantidad total puesta en juego), habiéndo formado $\mathrm{CaSO}_{4} .2 \mathrm{H}_{2} \mathrm{O}$ y ettringita en pequeña cantidad (figura 6), de acuerdo con las caracteristicas del cemento.

La formación de $\mathrm{Mg}(\mathrm{OH})_{2}(\mathrm{pK}=11,5)$, liberación de $\mathrm{Ca}(\mathrm{OH})_{2}(\mathrm{pK}=5,3)$, avance de las reacciones de hidratación y regulación del pH han hecho que se favorezca la obtención de $\mathrm{CaSO}_{4} .2 \mathrm{H}_{2} \mathrm{O}$ ( $\mathrm{pK}=4,6$ ), el cual reacciona con los aluminatos de calcio hidratados del cemento formando ettringita en pequeña cantidad; fenómenos que se han probado por DRX, de un modo especial en el cemento del lecho hidratado durante 7 días.

\subsection{Eyolución del pH y de la conductivida}

El pH de las distintas fracciones extraídas del agua desionizada viene regido por el equilibrio présents dans le ciment, étant fonction du degré d'hydratation et formation de nouveaux composants; ainsi, dans le cas du lit de ciment hydraté pendant 7 jours, $41 \%$-pour 194,69 Ides ces composants restent dans le ciment et $53 \%$-pour 140,19 I- dans le lit hydraté pendant 28 jours. L'extraction de ces composants diminue exponentiellement, comme dans le cas de Ca (II), avec le passage de l'eau déionisée.

Par contre, dans le cas de la solution de $\mathrm{Na}_{2} \mathrm{SO}_{4}$, lorsque les premières quantités de cette solution s'écoulent, la concentration de ions $\mathrm{SO}_{4}$ (II) y diminue (ils sont retenus par le lit du ciment) mais, par la suite, cette concentration se maintient pratiquement constante. En résumé, dès le commencement une partie de la concentration d'ions $\mathrm{SO}_{4}(\mathrm{II})$, en petite quantité et étant donné les caractéristiques structurelles du ciment, a été retenue par le ciment hydraté du lit, fondamentalement, sous forme de $\mathrm{CaSO}_{4} .2 \mathrm{H}_{2} \mathrm{O}$, ayant $\mathrm{Ca}(\mathrm{II})$-procédent $\mathrm{du}$ $\mathrm{Ca}(\mathrm{OH})_{2}-$. Par contre, ayant atteint l'équilibre et n'existant pas de $\mathrm{Ca}(\mathrm{II})<>\mathrm{Ca}(\mathrm{OH})_{2}$, puisqu'il s'est dissout, la formation de ce composant et de l'ettringite s'arrête, la solution de $\mathrm{Na}_{2} \mathrm{SO}_{4}$ agissant comme dissolvant des composants du ciment.

Dans les systèmes ciment-solution de sulfate de magnésium a lieu un procès analogue à celui de la solution de sulfate de sodium (la teneur en $\mathrm{SO}_{4}$ (II) diminue dans la première extraction et n'expérimente pas, par la suite, de variation); cependant, étant donné les caractéristiques de ces systèmes qui mettent en jeu des quantités plus grandes de $\mathrm{Ca}(\mathrm{II})$, d'après l'équilibre (B), les quantités d'ions $\mathrm{SO}_{4}$ (II), sont plus grandes (de l'ordre de 10-12\%, face à 6-7\% par rapport à la quantité totale mise en jeu), ayant formé $\mathrm{CaSO}_{4} .2 \mathrm{H}_{2} \mathrm{O}$ et ettringite en petite quantité (fig. 6) en accord avec les caracteristiques du ciment.

La formation de $\mathrm{Mg}(\mathrm{OH})_{2}(p K=11,5)$, la liberation de $\mathrm{Ca}(\mathrm{OH})_{2}(p K=5,3)$, l'avancement des réactions d'hydratation et la régulation du $\mathrm{pH}$ ont favorisé l'obtention de $\mathrm{Ca}_{2} \mathrm{SO}_{4} \cdot 2 \mathrm{H}_{2} \mathrm{O}$ ( $p K=4,6)$, qui réactionne avec les aluminates de calcium hydraté du ciment, produisant ettringite en petite quantité; phénomènes qui ont été prouvés par $D R X$, spécialement dans le ciment du lit hydraté pendant 7 jours.

\subsection{Evolution du $\mathrm{pH}$ et de la conductivité}

Le $\mathrm{pH}$ des différentes fractions extraites de l'eau déionisée est gouverné par l'équilibre (A). 
(A). En las primeras extracciones se produce un incremento brusco, llegando a alcanzar valos superiores a 12 , que permanecen prácticamente constantes en una primera etapa; a continuación, descienden dichos valores (son superiores a 9). La evolución del $\mathrm{pH}$ está de acuerdo con la cantidad de $\mathrm{Ca}(\mathrm{OH})_{2}$ disuelto, del mismo modo que en los sistemas cemento-disolución de sulfato de sodio.

En los sistemas cemento-disolución de sulfato de magnesio influye, además de los procesos anteriores, la reacción de precipitación del $\mathrm{Mg}(\mathrm{OH})_{2}$, manteniéndose en el entorno comprendido entre 10,6 y 10,7 a partir de las primeras extracciones, en donde se encuentra en valores del orden de 11,8.

En cuanto a la conductividad, que es función de los iones presentes en las disoluciones, viene regida, de un modo especial, por el equilibrio (A), experimentando un incremento brusco en las primeras extracciones $y$, a continuación, disminuye exponencialmente de modo análogo a como lo hace la cantidad de $\mathrm{Ca}(\mathrm{OH})_{2}$ disuelto. En los valores de la conductividad influyen, fundamentalmente, los iones $\mathrm{OH}$ (I) y $\mathrm{Ca}$ (II) en los primeros sistemas y, además, los iones $\mathrm{SO}_{4}$ (II) y $\mathrm{Na}$ (I) en los segundos y los iones $\mathrm{SO}_{4}$ (II) y Mg (II) en los terceros, cuyas conductividades iónicas a $25^{\circ} \mathrm{C}$, en ohm ${ }^{-1}$. $\mathrm{cm}^{2}$.equiv. ${ }^{-1}$, son: $\lambda \mathrm{OH}(\mathrm{l})=198,0$, $\lambda \mathrm{Ca}(\mathrm{II})=59,5, \lambda \mathrm{SO}_{4}(\mathrm{II})=80,0, \lambda \mathrm{Na}(\mathrm{I})=50,1$ $\lambda \mathrm{Mg}(\mathrm{II})=53,1$ (a dilución infinita).

\section{CONCLUSIONES}

\section{Primera}

$\mathrm{El} \mathrm{Ca}(\mathrm{OH})_{2}$ presente en los lechos de cemento hidratado, que procede de las reacciones de hidratación del cemento, se disuelve, parcialmente, en el agua desionizada que atraviesa dichos lechos y en menor grado en la disolución de sulfato de sodio; asimismo, en el agua desionizada se disuelven, parcialmente, los compuestos a base de sulfatos y de un modo especial la ettringita.

Las cantidades disueltas, que experimentan una disminución exponencial conforme aumenta el volumen de la disolución que atraviesa los lechos, son función del tiempo de hidratación-curado del cemento de los lechos, de las características de la disolución ( $\sin$ iones o con iones comunes y que reaccionan con otros iones) y del volumen de la disolución.
Dans les premières extractions un accroissement brusque se produit, pouvant atteindre des valeurs supérieures à 12, qui restent pratiquement constantes dans une première étape; de suite, elles descendent (elles sont supérieures à 9). L'évolution $d u p H$ est en accord avec la quantité de $\mathrm{Ca}(\mathrm{OH})_{2}$ dissout, de la même façon que dans les systèmes ciment-dissolution de sulfate de sodium.

Les systèmes ciment-solution de sulfate de magnésium, reçoit l'influence, en autre des procès précedents, de la réaction de précipitation de $\mathrm{Mg}(\mathrm{OH})_{2}$, qui maintient le $\mathrm{pH}$ dans la zone comprise entre 10,6 et 10,7 à parti des premières extractions, où il se trouve à environ 11,8

Quant à la conductivité, qui est fonction des ions présents dans les solutions, elle est gouvernée, spécialement, par l'éouilibre (A), subissant une augmentation brusque dans les premières extractions et, par la suite, diminuant exponentiellement d'une façon analogue à celle que subit la quantité de $\mathrm{Ca}(\mathrm{OH})_{2}$ dissout. Les valeurs de conductivité subissent fondamentalement l'influence des ions $\mathrm{OH}(\mathrm{I})$ et $\mathrm{Ca}$ (II) dans les premiers systèmes et, en plus, les ions $\mathrm{SO}_{4}(\mathrm{II})$ et $\mathrm{Na}$ (I) dans les secondes et les ions $\mathrm{SO}_{4}$ (II) et $\mathrm{Mg}$ (II) dans les troisièmes, dont les conductivités ioniques a $25^{\circ} \mathrm{C}$, en $\mathrm{ohm}^{-1} \cdot \mathrm{cm}^{2} \cdot$ equiv. $^{-1}$, sont:

$\lambda \mathrm{OH}(I)=198,0 ; \lambda \mathrm{Ca}(I I)=59,5 ; \lambda \mathrm{SO}_{4}(\mathrm{II})=$ $=80,0 ; \lambda \mathrm{Na}(\mathrm{I})=50,1$ et $\lambda \mathrm{Mg}(\mathrm{II})=53,1$

(à dilution infinie).

\section{CONCLUSIONE}

\section{Première}

Le $\mathrm{Ca}(\mathrm{OH})_{2}$ présent dans les lits de ciment hydraté, qui procédent des réactions d'hydratation du ciment, se dissout, partiellement, dans l'eau déionisée qui traverse ces lits et en plus petit degré dans la solution de sulfate de sodium; de même, les composants à base de sulfates et spécialement l'ettringite, se disolvent (partiellement) dans l'eau déionisée.

Les quantités dissoutes, qui expérimentent une diminution exponentielle en même temps que le volume de la solution qui traverse les lits augmente, sont fonction du temps d'hydratation-conservation du ciment des lits, des caractéristiques de la solution (sans ou avec ions, communs ou non, et qui réactionnent avec d'autres ions) et du volume de la solution. 


\section{Segunda}

Asimismo, dicho $\mathrm{Ca}(\mathrm{OH})_{2}$ se disuelve parcialmente en la disolución de sulfato de magnesio que atraviesa los lechos del cemento. Esta disolución del mencionado hidróxido se ve influenciada por la presencia de $\mathrm{Mg}$ (II) en la disolución, que precipita como brucita en los lechos, llegando a colmatarlos en determinados casos.

La evolución del contenido de Mg (II) en la disolución está íntimamente ligada a la del $\mathrm{Ca}$ (II) en dicha disolución y viceversa, de tal modo que cuando la concentración del uno aumenta la del otro disminuye.

El contenido de $\mathrm{Mg}$ (II) en la disolución desaparece total o parcialmente cuando atraviesa los lechos del cemento; totalmente cuando $\left[\mathrm{Ca}\right.$ (II)] $\geq 1,12 \times 10^{-2}$ y $\mathrm{pH} \geq 11,5$. Cuando hay $\mathrm{Mg}$ (II) en la disolución se cumple $[\mathrm{Ca}(\mathrm{II})]+[\mathrm{Mg}(\mathrm{II})]=$ cte $\left[\simeq 1,12 \times 10^{-2}\right.$ moles/litro $=$ concentración inicial de Mg (II)].

\section{Tercera}

Los iones $\mathrm{SO}_{4}$ (II) de las disoluciones de $\mathrm{Na}_{2} \mathrm{SO}_{4}$ y $\mathrm{MgSO}_{4}$ quedan retenidos, parcialmente, en los lechos de cemento, dando lugar a la formación de pequeñas cantidades de yeso y ettringita (mayores en el caso de la disolución de $\mathrm{MgSO}_{4}$ ); esta retención disminuye conforme aumenta el volumen de la disolución que ha atravesado los lechos, dependiendo, a su vez, del tiempo de curado del cemento.

\section{Cuarta}

La conductividad y el $\mathrm{pH}$ de las disoluciones que han atravesado los lechos de cemento hidratado, siguen una evolución análoga a la del $\mathrm{Ca}(\mathrm{OH})_{2}$ extraído, siendo función, de un modo especial, de la concentración de iones $\mathrm{OH}$ (I) presentes en las mencionadas disoluciones.

\section{Quinta}

Los compuestos cristalinos del cemento hidratado (ettringita, portlandita y calcita) y de la fracción no hidratada del clínker experimentan modificaciones considerables que dependen de la disolución (clase y volumen) y del tiempo de curado del cemento.

\section{Deuxième}

De même, ce $\mathrm{Ca}(\mathrm{OH})_{2}$ se dissout dans la solution de sulfate de magnésium qui traverse les lits de ciment. La solution de l'hydroxide mentionné est influencée par la présence de Mg (II) dans la solution, qui précipite comme brucite dans les lits, pouvant arriver à les colmater dans des cas détérminés.

L'évolution de la teneur en $\mathrm{Mg}$ (II) dans la solution est en rapport étroit avec celle du $\mathrm{Ca}$ (II) dans cette solution et viceversa, de telle façon que, lorsque la concentration de l'un augmente, celle de l'autre diminue.

La teneur en $M g$ (II) dans la solution disparait totalement ou partiellement lorsqu'il traverse les lits de ciment; totalement lorsque [Ca (II)] $\geq 1,12 \times 10^{-2}$ et $p H \geq 11,5$. Lorsqu'il y a $\mathrm{Mg}$ (II) dans la solution, il y a [Ca (II)] + $+[M g(I I)]=$ cte $\left[\simeq 1,12 \times 10^{-2}\right.$ moles/litre $=$ concentration initielle de $M g(I I)]$.

\section{Troisième}

Les ions $\mathrm{SO}_{4}$ (II) des solutions de $\mathrm{Na}_{2} \mathrm{SO}_{4}$ et $\mathrm{MgSO}_{4}$ sont retenues partiellement dans les lits de ciment, où a lieu la formation de petites quantités de gypse secondaire et d'ettringite (plus grandes dans le cas de la solution de $\mathrm{MgSO}_{4}$ ); cette rétention diminue en même temps que le volume de la solution qui a traversé les lits augmente, en dépendance, à son tour, du temps de conservation du ciment.

\section{Quatrième}

La conductivité et le $\mathrm{pH}$ des solutions qui ont traversé les lits de ciment hydraté poursuivent leur évolution, analogue à celle du $\mathrm{Ca}(\mathrm{OH})_{2}$ extrait, étant fonction, d'une façon spéciale, de la concentration d'ions $\mathrm{OH}(\mathrm{I})$ présents dans les solutions mentionées.

\section{Cinquième}

Les composants cristallins du ciment hydraté (ettringite, portlandite et calcite) et de la fraction non hydratée du clinker, expérimentent des changements considérables qui dépendent de la solution (classe et volume) et du temps de conservation du ciment. 
(1) GASPAR-TEBAR, D. SAGRERA-MORENO, J. L. y GONZALEZ-VILA, V. (1985): Contribución al estudio de la resistencia química de los cementos. Comportamiento mecánico de un cemento portland resistente al yeso ( $1 .^{\mathrm{a}}$ parte). Materiales de Construcción; abril, mayo, junio, 198, 31-41.

(2) GASPAR-TEBAR, D.; SAGRERA-MORENO, J. L. y GONZALEZ-VILA, V. (1985): Contribución al estudio de la resistencia química de los cementos. Comportamiento mecánico de un cemento portland resistente al yeso (2. ${ }^{\mathrm{a}}$ parte). Materiales de Construcción; octubre, noviembre, diciembre, 200, 49-86.

(3) GASPAR-TEBAR, D. et SAGRERA-MORENO, J. L. (1987): L'action de l'eau de mer sur un ciment portland de haute resistance initielle et sur un ciment portland resistant aux sulfates: influene de l'addition de laitier. Etude de la concentration ionique. Materiales de Construcción, abril, mayo, junio, 206, 27-42.

(4) GASPAR-TEBAR, D. et SAGRERA-MORENO, J. L. (1987): L'action de l'eau de mer sur un ciment portland de haute resistance initielle, sur un ciment portland resistant aux sulfates et sur un ciment portland ordinaire: influence de l'addition de laitier. Etude par DRX. Materiales de Construcción, julio, agosto, septiembre, 207, 13-35.

(5) BICZOK, I. (1972): La corrosión del hormigón y su protección. Edic. Urmo; Bilbao, pág. 211.

(6) DURIEZ, M. et ARRAMBADI, J. (1961): Nouveau traité de Matériaux de Construction; Tome 1. Edit. Dunod; Paris, págs. 489-490.

(7) ALEGRE, R. (1978): Comportement des ciments en milieu agressif. Annales de l'Institut Technique du Bâtiment et des Travaux Publics (Serie: Sols et Fundations, n.0 159), 364, pág. 78.

(8) DREYFUS, J. (1960): La Chimie des Ciments; Tome 1. Edit. Eyrolles; París, pág. 41.

(9) TAYLOR, H. F. W. (1964): The Chemistry of Cements; vol. 1. Edit. Acad. Press Inc.; London, pág. 228.

(10) LEA, F. M. y DESCH, E. H. (1960): Química del Cemento y del Hormigón. Edit. Tipografía Artística. Madrid, pág. 228.

(11) BOGUE, R. H. (1962): La Química del Cemento Portland. Edit. Dossat. Madrid, pág. 600.

\section{AGRADECIMIENTOS}

Nuestro más sincero agradecimiento a las personas del Equipo de "Durabilidad" del IETcc: Amalia Rodríguez Pereíra, Lucila López Solana, M. ${ }^{a}$ Soledad Cid Fernández, Felipe Cantero Palacios y Manuel Cantero Palacios por su valiosa colaboración en la realización de este trabajo.

\section{REMERCIEMENTS}

Nous voudrons exprimer nôtre plus vive reconnaissance aux membres de l'El "Durabilidad" de I'IETcc: Amalia Rodríguez Pereíra, Lucila López Solana, M. ${ }^{a}$ Soledad Cid Fernández, Felipe Cantero Palacios y Manuel Cantero Palacios pour l'aide prêtée dans la réalisation de ce travail.

\section{nota importante}

Con este número finaliza la suscripción concertada con esta Revista.

En vista de la respuesta obtenida por parte de nuestros lectores, y en atención a los mismos, los precios no sufren alteración con respecto a 1987, es decir, que los suscriptores de España y Extranjero en 1988 abonarán los importes ya establecidos en el año que termina. 